\title{
EL VIAJE A PORTUGAL \\ DE LOS EMBAJADORES DE FEDERICO III EN EL RELATO DE LANCKMANN Y DE OTROS CRONISTAS
}

\author{
KLAUS HERBERS ${ }^{1}$
}

Fecha de recepción: octubre 2001.

Fecha de aceptación y versión final: diciembre 2001.

Resumen: El artículo analiza el relato de Nicolás Lanckmann y Jacobo Motz sobre el viaje que éstos emprendieron hacia Lisboa en 1451 para llevar a la futura esposa de Federico III, Leonor de Portugal, a Roma. Se destaca el papel de los salvoconductos y de las tradiciones jacobeas, y se estudian asimismo los motivos para elegir la ruta por el norte de España y el desvio hacia Santiago de Compostela durante el Año Santo compostelano. La difusión del relato en los siglos XV y XVI subraya como se formó también en los países de lengua alemana una idea de la Península Ibérica

Palabras clave: Emperador Federico III, Leonor de Portugal, Literatura de viajes, Santiago de Compostela.

Abstract: The article analyses the report by Nicolas Lanckmann and Jacob Motz of the trip they undertook in 1451 to Lisbon in order to accompany Eleonore of Portugal, future wife to Frederic III, to Rome. Special importance is given to the role safe-conducts and Jacobean traditions play in the text, and the motives for taking the Northern route through the Iberian Peninsula and for diverting to Santiago de Compostela during the time of the "Año Santo" are examined. The report's wide circulation during the XVth and XVIth centuries underlines the ways one formed an image of the Iberian Peninsula in the countries of German tongue. de Compostela.

Key-words: Emperor Frederic III, Eleonora of Portugal, Travel Literature, Santiago

${ }^{1}$ Catedrático de Historia Medieval y de Ciencias Auxiliares, Universität Erlangen-Nürnberg (República Federal de Alemania).

«Anuario de Estudios Medievales», 32/1 (2002), pp. 183-198.- ISSN 0066-5061. 
I. Introducción.- II. La misión diplomática deNicolás Lankmann y Jacobo Motz.- III. El viaje a Portugal por Compostela en el relato de Lanckmann y otras fuentes.- IV. Los viajes y las peregrinaciones en los relatos: 1 . Recepción de forasteros y salvoconductos. 2. Tradiciones jacobeas y de peregrinos. 3. El rodeo por Santiago.- V. Conclusión.

\section{INTRODUCCIÓN}

Consequenter itinerando venerunt... ad locum Sancti Iacobi, et eadem ciuitas dicitur Campistella. Ibique pro quiete et deuotione usque in tertium diem moram fecerunt et missas celebrauerunt - "En el curso del viaje llegaron luego... al lugar de Santiago, a la ciudad que lleva el nombre de Compostela. Ahí permanecieron tres días para descansar y por razones de devoción y fueron a misa" ${ }^{2}$. Esto es todo lo tocante a la ciudad gallega que menciona Nicolás Lanckmann en el relato que escribió luego del viaje que emprendiera a la Península Ibérica con Jacobo Motz. El fin de este viaje era llevar a efecto por procuración el casamiento de la infanta Leonor de Portugal con el rey de Alemania Federico III y escortar a la princesa a Italia. El texto de Lanckmann seguramente lo conocerá mejor su último editor, Aires Nascimento; es por eso que sólo quisiera presentar algunos aspectos que guardan relación con las peregrinaciones a Compostela y analizar el viaje de los dos embajadores en un contexto más amplio, el de los peregrinos y viajeros ultrapirenaicos que recorrieron España y Portugal a mediados del siglo XV. Se ha publicado mucho sobre los viajes y los respectivos relatos de peregrinos jacobeos que también visitaron Portugal ${ }^{3}$. La mayoría de las

${ }^{2}$ Aires A. NASCIMENTO (con la colaboración de Maria J. BRANCO y Maria de Lurdes RoSA), Leonor de Portugal, imperatriz da Alemanha. Diário de Viagem do Embaixador Nicolau Lanckmann de Valckenstein, Lisboa, 1992, p. 24 (agradezco a la Srta. Sofia Seeger, Erlangen, que me procurase la edición y también por su ayuda al preparar esta ponencia).

${ }^{3} \mathrm{Cf}$. la documentación sobre estos viajes en Raymond FOULCHÉ-DELBOSC, Bibliographie des voyages en Espagne et en Portugal, "Revue Hispanique" 3 (1896), pp. 1-373; Arturo FARINELLI, Viajes por España y Portugal desde la Edad Media hasta el siglo XX, 3 vol., Roma, 1942 ("Reale Accademia d'Italia, Studi e Documenti" 11); José GARCÍA MERCADAL, Viajes de extranjeros por España y Portugal desde los tiempos más remotos, hasta fines del siglo XVI, Madrid, 1952. Cf. también Ilja MIECK, Les témoignages oculaires du pèlerinage à Saint-Jacques de Compostelle. Etude bibliographique (du XII au XVII siècle), "Compostellanum" 22 (1977), pp. 3-32; Ursula 
personas provenientes de Europa Central que fueron a este país pasando por Santiago -por ejemplo, León de Rožmital, Nicolás de Popplau o Jerónimo Münzer y más tarde Lucas Rem, entre otros- también perseguía otras intenciones ajenas al peregrinaje. Entre éstas se pueden mencionar negocios, misiones diplomáticas, así como una cierta curiosidad etnográfica ${ }^{4}$.

\section{LA MISIÓN DIPLOMÁTICA DE NICOLÁS LANCKMANN Y JACOBO MOTZ}

En lo que se refiere a su objetivo, el viaje de Lanckmann y Motz difiere de otros. Federico III, rey de Alemania perteneciente a la dinastía de los Habsburgo, tenía bajo su tutela a su sobrino Ladislao Póstumo, heredero al trono de Bohemia y Hungría. En la corte de Borgoña, surgió en 1447 la idea de que Ladislao y su tío se casaran con dos infantas portuguesas. La carta destinada al canciller de Federico que contiene la propuesta no menciona el nombre de las princesas, pero sí su edad, por lo que es lícito suponer que se trata de Juana y Leonor ${ }^{\text {. }}$.

En aquel entonces, Federico ya tenía 32 años. El que un rey siguiera soltero a esa edad no era nada habitual. Varios proyectos de matrimonio anteriores habían fracasado, aparentemente, por rechazar el soberano a las diferentes mujeres, de verlas. De esto habría que deducir que Federico quería

GANZ-BLÄTTLER, Andacht und Abenteuer. Berichte europäischer Jerusalem- und Santiago-Pilger (1320-1520), 2a edición, Tubinga, 1991 ("Jakobus-Studien" 4); Werner PARAVICINI y Christian HALM, Europäische Reiseberichte des späten Mittelalters, parte I: Deutsche Reiseberichte, Francfort del Meno et al., 1994 (Kieler Werkstücke D, 5), pp. 7-15; Klaus HERBERS y Robert PLÖTZ, Nach Santiago zogen sie. Berichte von Pilgerfahrten ans "Ende der Welt", Munich, 1996 (dtv 4718), con versión castellana: Caminaron a Santiago. Relatos de peregrinaciones al "fin del mundo", Santiago de Compostela, 1998 (publicado en 1999).

${ }^{4}$ Cf. Klaus HERBERS, Spanienreisen im Mittelalter - unbekannte und neue Welten, "Das Mittelalter" 3/2 (1998): "Fernreisen im Mittelalter", ed. Folker REICHERT (publicado en 1999), pp. 81-106. Sobre el viaje de Popplau y de Münzer, cf. también: IDEM, Kulturtransfer durch Reisende? Schlesische und andere Westeuropa-Reisende im 15. Jahrhundert (por parecer en "Die Bedeutung der Jagiellonen für Kunst und Kultur Mitteleuropas (1450-1550)" e IDEM, 'Murcia ist so groß wie Nürnberg' - Nürnberg und Nürnberger auf der Iberischen Halbinsel: Eindrücke und Wechselbeziehungen, en "Nürnberg - europäische Stadt in Mittelalter und Neuzeit", ed. Helmut NeUHAUS, Neustadt an der Aisch, 2000 (Nürnberger Forschungen 29), pp. 151-183.

${ }^{5} \mathrm{La}$ carta está publicada en E. BIRK, Actenstücke, Memoriale Adrians van der Ee, 1447, $\mathrm{n}^{\circ}$ X, p. 259. Cf. Friedrich vON KRONES, Leonor von Portugal: Gemahlin Kaiser Friedrichs III., des steirischen Habsburgers (1436-1467), "Mittheilungen des Historischen Vereins für Steiermark", 49 (1901), pp. 53-120, esp. p. 59. 
casarse con una mujer hermosa. Como en esa época era usual que personas de la alta nobleza se casaran con personas de regiones remotas, existía incluso la costumbre de pedir retratos de las candidatas ${ }^{6}$. Esta costumbre la siguió también Federico, el cual en 1448 envió a dos de sus consejeros y un pintor a Portugal con el encargo de volver con un retrato de Leonor. A su regreso, los embajadores hablaron en muy buenos términos de la joven y resaltaron su belleza, después de lo cual Federico decidió entablar las negociaciones para el matrimonio.

Mientras tanto, había surgido otro aspirante a la mano de Leonor, el delfín, el futuro Luis XI de Francia. Sin embargo, al final la infanta se decidiría por Federico, esto es, por el futuro emperador.

Las negociaciones se llevaron a cabo en 1450 por medio de Alfonso $\mathrm{V}$ de Aragón, tío de Leonor. En diciembre del mismo año, en la corte del rey de Aragón en Nápoles, se fijaron las condiciones del casamiento: las sumas a pagar por las dos partes, las fechas y el camino a tomar por los embajadores de Federico, que irían a buscar a la novia. Las bodas se celebrarían por procuración en Lisboa; luego, las nupcias solemnes tendrían lugar en Roma, en presencia del $\mathrm{Papa}^{7}$.

Ya mencionamos que el viaje de los dos enviados, en este contexto, nos interesa sobre todo en lo que atañe a la visita de Santiago de Compostela. Existen diferentes fuentes que tratan de este viaje, sin embargo, el relato de Lanckmann ${ }^{8}$ mismo resulta de particular interés. La obra fue traducida al alemán. Tanto la versión latina original como la alemana fueron impresas en

${ }^{6}$ Sobre Leonor, cf. en general: KRONES, Leonor von Portugal (cf. nota 5); Antonia ZIERL, Kaiserin Eleonore und ihr Kreis: Eine Biographie (1436-67), Viena, 1966 (tesis de doctorado dactilográfica); L. CORDEIRO, Uma Sobrinha do Infante Imperatriz da Allemanha e Rainha da Hungria, Lisboa, 1894 (Portugueses fóra de Portugal); Bettina Antonia HANREICH, D, Leonor de Portugal, esposa do imperador Frederico III (1436-1467), en "Relações entre a Áustria e Portugal. Testemunhos históricos e culturais", ed. Ludwig SCHEIDL y José A. PALMA CAETANO, Coimbra, 1985, pp. 3-27; Eberhard HoLTZ, Eine Portugiesin in Österreich-Eleonore, Gemahlin Kaiser Friedrichs III., en "Fürstinnen und Städterinnen. Frauen im Mittelalter", ed. Gerald BEYREUTHER, Barbara PäTZOLD y Erika UITZ, Friburgo de Brisgovia, Basilea, Viena, 1993, pp. 255-282; Bettina PFERSCHY-MALECZEK, Kaiserin Eleonore, en "Frauen des Mittelalters in Lebensbildern", ed. Karl Rudolf SCHNITH, Graz, Viena, Colonia, 1997, pp. 420-446.

${ }^{7}$ Sobre los viajes de matrimonio, cf. Karl-Heinz SPIEß, Unterwegs zu einem fremden Ehemann. Brautfahrt und Ehe in europäischen Fürstenhäusern des Spätmittelalters, en "Fremdheit und Reisen im Mittelalter", ed. Irene ERFEN e IDEM, Stuttgart, 1997 (Mittelalterzentrum Greifswald), pp. 17-36, esp. p. 35.

${ }^{8} \mathrm{Cf}$. la nueva edición con traducción portuguesa de Aires A. NASCIMENTO (cf. nota 2), además de las ediciones anteriores mencionadas en PARAVICINI/HALM, Deutsche Reiseberichte (cf. nota 3), p. 120 
1503. En 1514, el texto sirvió de base a Max Treitzsauerwein para la redacción de su "Weißkunig" (manuscrito A), escrito en honor del hijo de Federico III, Maximiliano I ${ }^{9}$. Otro testimonio que hasta el día de hoy se ha tenido poco en cuenta es el relato de Cristóbal Ungnad ${ }^{10}$. Una fuente portuguesa la constituye la "Crónica de Alfonso V"11. En cuanto a los oratores procuratores del viaje, eran sacerdotes ambos; Jacobo Motz era bachiller en teología y confesor de Federico III, Lanckmann, capellán del rey.

\section{El VIAJE A PORTUGAL POR COMPOSTELA \\ EN EL RELATO DE LANCKMANN Y OTRAS FUENTES}

Los embajadores del rey recorrieron, pues, en parte, caminos jacobeos, para llegar a Portugal — como ha quedado plasmado en la obra de Lanckmann. ¿En qué medida su itinerario corresponde al de otros viajeros de la época? ¿Cuán presentes están las tradiciones jacobeas en el texto?

El texto empieza con la intitulatio de Federico; sigue después la descripción del trayecto de Lanckmann y Motz. Los dos embajadores pasaron por Ginebra y cruzaron el Delfinado, donde visitaron la Gran Cartuja y el monasterio de S. Antonio. Luego recorrieron el Languedoc y se detuvieron en Montpellier, ciudad y universidad de médicos. Pensaban pasar por Tolosa y Roncesvalles, lugares en que Carlomagno y Roldán vencieran al "rey de

${ }^{9} \mathrm{Cf}$. Erwin Koller, Die Verheiratung Eleonores von Portugal mit Kaiser Friedrich III. in zeitgenössischen Berichten, en "Portugal-Alemanha-África. Do Imperialismo Colonial ao Imperialismo Político, Actas do IV Encontro Luso-Alemão", ed. Antonio H. DE OLIVEIRA MARQUES, Alfred OpITZ y Fernando ClarA, Lisboa, 1996, pp. 43-56, esp. p. 45. Cf. Erwin KOLLER, O Portugal, o Portugal, wie gar ain guets kunigreich bist du! Die Brautwerbungsbotschaft Friedrichs III. im Weißkunig, en "Literatur und Sprache in Tirol. Von den Anfängen bis zum 16. Jahrhundert", ed. Michael GEBHARDT und Max SILLER, Innsbruck, 1996 (SchlernSchriften 301), pp. 293-321. Cf. también Karl RuDOLF, 'Das gemäl ist also recht'. Die Zeichnungen zum 'Weißkunig' Maximilians I. des Vaticanus Latinus 8570, "Römische Historische Mitteilungen" 22 (1980), pp. 167-205.

${ }^{10} \mathrm{Cf}$. KolLer, Verheiratung Eleonores (cf. nota 9), p. 45 con nota 8. Lanckmann conoció a Ungnad en Lisboa, puesto que éste también asistió a las ceremonias que tuvieron lugar con motivo del casamiento de Leonor y luego formó parte de la delegación que condujo a la infanta a Italia. Cf. NASCIMENTO, Leonor de Portugal (cf. nota 2), pp. 40 y 52.

${ }^{11}$ Cf. Rui de PINA, Crónicas de Rui de Pina, ed. Manuel Lopes De Almeida, Oporto, 1977. Sobre la documentación concerniente al casamiento y a las ceremonias que tuvieron lugar en Roma, cf. Achim Thomas HACK, Das Empfangszeremoniell bei mittelalterlichen Papst-KaiserTreffen, Colonia et al., 1999 (Forschungen zur Kaiser- und Papstgeschichte des Mittelalters 18), pp. $14-80$ y $670-680$. 
Granada" (sic), pero aquella ruta era insegura debido al peligro que representaban los Armagnac, razón por la cual finalmente optaron por ir a Cataluña por Narbona y Perpiñán. En Barcelona, la illustrissima domina Arragonum et Sicilie regina les dio un salvoconducto, cuyo texto Lanckmann incluye en su relato ${ }^{12}$. Los dos sacerdotes cabalgaron después por Cataluña, una región fértil y rica, aunque peligrosa, porque sus habitantes se dedicaban a hacer la guerra y a la rapiña.

Enseguida recorrieron Aragón. Lanckmann describe un poco más detenidamente la ciudad de Zaragoza, mencionando tres mezquitas y una escuela para jóvenes musulmanes. En esta ciudad se habrían observado tres días festivos por semana: el viernes, el sábado y el domingo, días sagrados a los sarracenos, judíos y cristianos, respectivamente. A los embajadores se les entregó además un segundo salvoconducto. Después de visitar Lleida con su universidad, llegaron a Navarra.

Santo Domingo de la Calzada consigue captar el interés de Lanckmann. Casi la mitad del tercer capítulo trata de esa ciudad. El autor resalta dos aspectos: la catedral en que se exhiben gallinas, en recuerdo del famoso milagro jacobeo del ahorcado, y una secta. Los miembros de esta última habrían sido quemados, por pretender llevar una vida a semejanza de la de Cristo. Uno de ellos se habría hecho llamar Cristo, los otros se habrían considerado sus apóstoles y discípulos. En Burgos, el capitán les concedió otro salvoconducto. Camino a León, se encontraron con hombres armados que a la fuerza los llevaron ante la presencia del monarca castellano, que estaba en guerra con el rey de Granada. El soberano les otorgó otro salvoconducto en la lengua de su país. León -cuenta Lanckmann- era sede episcopal y contaba con una universidad. Los religiosos llegaron luego a Astorga, donde se quedaron por un día y una noche, pues un caballero sevillano había atacado al conde Alvaro de Luna, duque de Galicia.

$\mathrm{Al}$ día siguiente, Lanckmann y Motz se unieron more peregrinorum a un grupo de peregrinos provenientes de diferentes países y regiones, para seguir viaje a Galicia. En el Rabanal, los asaltaron trescientos hombres, algunos de los cuales iban montados. Éstos hicieron prisionero al grupo. A Lanckmann y Motz les quitaron la ropa, el dinero y un caballo. Los embajadores besaron los pies al capitán y le enseñaron sus salvoconductos.

${ }^{12}$ Cf. Nascimento, Leonor de Portugal (cf. nota 2), pp. 18-20. 
Después imploraron la ayuda de Santiago y de San Jorge. Finalmente, mostraron al jefe de los asaltantes la carta de Federico III.

Los bandidos soltaron a los peregrinos. Los dos religiosos pernoctaron en un pueblo sin mayor interés. Al día siguiente, fiesta de San Ulrico - esto es, el 4 de julio-, siguieron en dirección a Galicia, pasando por Ponferrada. En esta ciudad, un conde les otorgó un préstamo contra acuse de recibo. Le devolvieron el dinero en Santiago de Compostela, donde existía una sucursal del banco de Cosme de Médicis. Lanckmann y Motz se quedaron tres días en la ciudad gallega, antes de proseguir viaje, rumbo a Portugal. Pasaron por Padrón, adonde el apóstol Santiago habría llegado en una barca de piedra y donde habría hecho muchos milagros. La barca habría sido encontrada cerca de la costa y ahora - relata Lanckmann - se exhibía a los peregrinos. Después, propter metum raptorum, los embajadores se asociaron a un grupo de peregrinos irlandeses, con los cuales fueron a Finisterre y visitaron la capilla de Santa María. Luego un guía los llevó a Tuy, ciudad a orillas del Miño, que separa Galicia de Portugal.

\section{LOS VIAJES Y LAS PEREGRINACIONES EN LOS RELATOS}

No quisiera detenerme en las ceremonias que tuvieron lugar en Portugal ni en el choque de la cultura portuguesa con la alemana; quisiera destacar más bien algunos aspectos del viaje y compararlo con otros viajes de nobles por esa misma época. Esos viajes han sido investigados, con interesantes resultados en lo que respecta al contacto de culturas diferentes ${ }^{13} \mathrm{o}$ al ritual observado con ocasión del encuentro de personas pertenecientes a culturas diferentes ${ }^{14}$.

${ }^{13} \mathrm{Cf}$. SPIEß, Unterwegs (cf. nota 7), pp. 31-32.

${ }^{14} \mathrm{Cf}$. sobre la noche de bodas en Nápoles y el relato de Eneas Silvio Piccolomini (versión alemana: Die Geschichte Kaiser Friedrichs III., trad. por Theodor ILGEN, vol. II, $2^{\mathrm{a}}$ edición, Lipsia, 1940 ("Die Geschichtsschreiber der deutschen Vorzeit" 89/2), pp. 95-97; y el estudio de M. SCHRÖTER, Zur Intimisierung der Hochzeitsnacht im 16. Jahrhundert, en "Ordnung und Lust. Bilder von Liebe, Ehe und Sexualität in Spätmittelalter und früher Neuzeit", ed. Hans-Jürgen BACHORSKI, Tréveris, 1991 ("Literatur, Imagination, Realität" 1), pp. 359-414. 


\section{Recepción de forasteros y salvoconductos}

Llama la atención el hincapié que hace Lanckmann en los salvoconductos y en la forma de adquirirlos. Estos documentos eran de fundamental importancia para ser bien recibido en los lugares que se visitaban. La red de contactos entre los nobles y las recomendaciones resultan de gran interés para los investigadores de la nobleza en los siglos XV y XVI ${ }^{15}$. Las recomendaciones también sirven al momento de un asalto ${ }^{16}$, aunque no solamente por su contenido: no se escribían tan sólo en latín, sino también en otros idiomas, y no siempre las personas entre cuyas manos caían podían leerlas. Según consta del relato de Lanckmann, el salvoconducto de Federico ${ }^{17}$ fue sobre todo lo que los salvó a él y a su compañero. Tal vez el sello impresionara a los bandidos, que se quedaron con el documento.

\section{Tradiciones jacobeas y de peregrinación}

En el relato de Lanckmann, aparecen varias tradiciones jacobeas. El itinerario por el Sur de Alemania y por Saboya, pasando por Ginebra y por el monasterio de S. Antonio, coincide con el descrito en otras fuentes, por ejemplo en la guía de Hermannus Künig de Vach de finales del siglo XV, que denomina este camino "Vía superior" (Oberstraße ${ }^{18}$ ) y que resalta la importancia de esta abadía. Lanckmann menciona además las tradiciones relativas a Roncesvalles y habla de Carlomagno y Roldán, los cuales habrían

\footnotetext{
${ }^{15}$ Sobre todo Werner Paravicini ha resaltado la existencia de una red de contactos entre los nobles a escala europea: cf. por exemplo IDEM, Die Preußenreisen des europäischen Adels, partes I-II ("Beihefte der Francia" 17/1-2, Sigmaringen, 1989-1995); IDEM, Von der Heidenfahrt zur Kavalierstour. Über Motive und Formen adligen Reisens im späten Mittelalter, en "Wissensliteratur im Mittelalter und in der Frühen Neuzeit. Bedingungen, Typen, Publikum, Sprache (Wissensliteratur im Mittelalter" 13), Wiesbaden, 1993, pp. 91-130.

${ }^{16} \mathrm{Cf}$. Werner PARAvicini, Fürschriften und Testimonia. Der Dokumentationskreislauf der spätmittelalterlichen Adelsreise am Beispiel des kastilischen Ritters Alfonso Mudarra 1411-1412, en "Studien zum 15. Jahrhundert, Festschrift Erich Meuthen", ed. Johannes HELMRATH y Heribert MÜLLER, Munich, 1994, pp. 903-926.

${ }^{17}$ En el texto en latín, aparecen litterae y littera passus. NASCIMENTO, Leonor de Portugal (cf. nota 2), pp. 22 y 24 .

${ }^{18} \mathrm{Cf}$. el facsímil A peregrinaxe e o Camiño a Santiago. Hermannus Künig de Vach, ed. Klaus HERBERS y Robert PLÖTZ, Santiago de Compostela, 1999, p. 21, trad. gallega, p. 44; cf. además la traducción española de Hermannus Künig de Vach en HERBERS y PLÖTZ, Caminaron a Santiago (cf. nota 3), p. 206.
} 
luchado contra los paganos a las órdenes del rey de Granada. Sin embargo, ya se mencionó que los embajadores no pasaron por el paso de Roncesvalles, por los peligros que acechaban en las vías, especialmente por parte de los Armagnac, una confraternitas bellatorum, sino que se unieron a un grupo de mercaderes, para seguir camino a Cataluña ${ }^{19}$.

Al hablar de Santo Domingo, Lanckmann menciona otra tradición jacobea que aparecería en el Pasionario: el milagro del ahorcado. Se trata presumiblemente de "Das alte Pasional ${ }^{20}$. Resulta interesante que al autor interese más la secta que el milagro jacobeo ${ }^{21}$. Luego los embajadores son atacados en el monte Rabanal. El suceso hace pensar en Hermannus Künig, que en su guía aconsejaba a los peregrinos: huotte dich vor der rabenel ist myn rat - "ten cuidado con el Rabenel, éste es mi consejo"22. También la canción alemana de los peregrinos recomienda estar alerta en los montes, entre otros en el Rabanal ${ }^{23}$.

En lo que a Compostela se refiere, el relato de Lanckmann no entra en detalles. Él y su compañero de viajes se quedaron tres días en dicha ciudad, sin embargo sólo trata tradiciones jacobeas en los pasajes relativos a Padrón, Finisterre y la capilla de Santa María (Mugia).

\section{El desvio hacia Santiago}

¿A qué se debe el que los embajadores de Federico III escogieran la ruta por Santiago, ciudad que Lanckmann apenas describe? ¿Estaba previsto tomar ese camino desde un comienzo? No lo sabemos a ciencia cierta. ¿Habrá

${ }^{19} \mathrm{Vgl}$. NASCIMENTO, Leonor de Portugal (cf. nota 2), p. 8; en lo referente a los mercaderes de Europa Central en camino hacia la Peninsula Ibérica, cf. Hermann KELLENBENZ, Die fremden Kaufleute auf der Iberischen Halbinsel vom 15. Jahrhundert bis zum Ende des 16. Jahrhunderts, en "Fremde Kaufleute auf der Iberischen Halbinsel", ed. IDEM, Colonia, Viena, 1970, pp. 265376, sobre todo pp. 301-328 (y las otras contribuciones en el mismo volumen).

${ }^{20}$ Werner Williams, 'Di grôsten Zeichen di kein heilige getun mac di tut dirre heilige'. $\mathrm{Zu}$ den deutschen Jakobslegenden, en "Der Jakobuskult in Süddeutschland", ed. Dieter R. BAUER y Klaus HERBERS, Tubinga, 1995 ("Jakobus-Studien" 7), pp. 233-248, p. 232, cf. sobre la leyenda de Sto. Domingo en "Das alte Passional", p. 238.

${ }^{21}$ NASCIMENTO, Leonor de Portugal (cf. nota 2), p. 22.

${ }^{22} \mathrm{~V} .481$, cf. el facsímil de la edición de 1495, HERBERS/PlÖTZ, Peregrinaxe (cf. nota 18), p. 36, trad. gallega, p. 62; cf. la traducción al español en HERBERS/PLÖTZ, Caminaron a Santiago (cf. nota 3), p. 206.

${ }^{23}$ Herbers/PlötZ, Caminaron a Santiago (cf. nota 3), p. 162. 
querido Jacobo Motz visitar la tumba de Santiago, cuyo nombre llevaba? Otros viajeros optaron por seguir itinerarios diferentes: Jerónimo Münzer recorrió toda la Península Ibérica y llegó a Santiago desde Portugal ${ }^{24}$. Nicolás de Popplau fue de Inglaterra a Portugal, pasando por La Coruña y Santiago, mientras que León de Rožmital llegó a Portugal siguiendo el curso del Duero y de ese país se dirigió a la ciudad del Apóstol, para luego volver a él. Desde Alemania y Francia, dos caminos principales conducían a España, el por Irún y el por Barcelona, según podemos colegir del Itinerario de Brujas y, sobre todo, del mapa de Erhardo Etzlaub de 1501 y del "Reisbüchlin" de Jorge Gail $^{25}$.

¿Por qué Lanckmann y Motz no siguieron el curso del Duero, tal como lo haría, por ejemplo, en 1466, León de Rožmital, o no tomaron otro camino más directo? Llama la atención que, para mayor seguridad, buscaran siempre la compañía de otros viajeros ${ }^{26}$. Evitaron cruzar por el paso de Roncesvalles, por el peligro que suponía la acción armada de los Armagnac y llegaron a España por Perpiñán y Gerona, junto con un grupo de mercaderes ${ }^{27}$. De este modo, la situación política del momento determinó su ruta. Así, fueron, por ejemplo, a la corte aragonesa. Cabe subrayar aquí que Alfonso V de Aragón, tío de Leonor, había sido uno de los principales promotores del casamiento entre Federico y su sobrina y que el convenio de matrimonio había sido firmado en diciembre del año anterior en su corte en Nápoles. Parece que los dos enviados no tenían pensado pedir audiencia al rey

${ }^{24}$ Cf. HERBERS, Murcia (cf. nota 4), p. 155 (mapa con el itinerario de Münzer).

${ }^{25}$ Itinerario de Brujas: Le livre de la description des pays de Gilles le Bouvier, dit Berry, suivi de l'Itineraire Brugeois, ed. E.-T. HAMY, París, 1908; sobre Etzlaub cf. Herbert KRÜGER: Erhard Etzlaubs Romweg-Karte, eine Pilgerstraßen-Karte zum Heiligen Jahr 1500, en "Mitteilungen der Geographischen Gesellschaft Wien", 92 (1950), pp. 202-204; cf. IDEM, Des Nürnberger Meisters Erhard Etzlaub älteste Straßenkarten von Deutschland, en "Jahrbuch für Fränkische Landesforschung”, 18 (1958), pp. 1-286, 188-193 y 278; sobre Jorge Gail cf. Herbert KRÜGER, Das älteste deutsche Routenhandbuch, Jörg Gails >Raißbü̈chlin`. Mit 6 Routenkarten und 272 Originalseiten im Faksimile, Graz, 1974, pp. 292-302. Cf. también Carlos GIL y João RODRIGUES, Pelos Caminhos de Santiago. Itinerarios portugueses para Compostela, Lisboa, 1990.

${ }^{26} \mathrm{La}$ búsqueda de compañeros de viaje para correr menos peligro es un elemento recurrente en las fuentes, que aparece, por ejemplo, en la carta de Hermannus de Tournai a Anselmo y Gualterio de Laon de 1143. Cf. Epistola Hermanni abbatis S. Martini Tornacensis de corpore S. Vincentii Diaconi, ed. K. DE SMEDT, W. VAN HOOF y J. DE BACKER, “Analecta Bollandiana", 2 (1883), pp. 243-246, esp. p. 246.

${ }^{27}$ Kellendenz, Deutsche Kaufleute (cf. nota 19), p. 273 y HerBers, Murcia (cf. nota 4), p. 156 . 
de Castilla, Juan II (1406/19-1454), pero, al ser apresados por los hombres de éste, fueron llevados por la fuerza ante su presencia. En Astorga tuvieron que detenerse por haber sido atacado el conde Alvaro de Luna.

Fueran los que fueran los motivos que condicionaron la elección de la ruta, los dos enviados aprovecharon el viaje para visitar la tumba del Apóstol. Lanckmann menciona su devoción al Santo y la de su acompañante; al momento de caer presos en manos de los bandidos del Rabanal, ambos imploran la ayuda de Santiago. Rui de Pina lo dice explícitamente: los embajadores fueron en romería a Santiago ${ }^{28}$. Luego de verse desprovistos de todas sus pertenencias en el Rabanal y de recibir el préstamo del conde en Ponferrada, la visita a Santiago se tornó obligatoria, puesto que ahí se encontraba la sucursal de los Médicis que les permitía saldar sus deudas. Al parecer, esta dependencia era la única en todo el Noroeste de España ${ }^{29}$.

Si bien Lanckmann muestra interés por diferentes fenómenos, no deja de notar aspectos relacionados con el culto y la religión. Así, al hablar de la Catedral de Lisboa, menciona dos veces que en ella reposa el cuerpo de S. Vicente. Cuenta además que al partir de Portugal, Leonor se despidió de este santo, bajo cuyo patronato había nacido y había sido bautizada ${ }^{30}$. El interés de este pasaje reside en que representa un testimonio de la veneración de los reyes portugueses al diácono de Zaragoza. De esta manera, el relato del sacerdote alemán constituye una fuente para estudiar el culto a los santos a mediados del siglo XV. Lanckmann también trata las "cruzadas" portuguesas en el Norte de África ${ }^{31}$.

El día de $\mathrm{S}$. Ulrico, esto es, el 4 de julio, los dos enviados llegaron a Galicia ${ }^{32}$. Al hablar de Portugal, el autor menciona que él y su acompañante estuvieron en Santiago el 20 de julio. Llama la atención que no se quedaran ahí hasta el 25 de julio, fiesta del Santo. Nascimento asoció la visita a Padrón

${ }^{28}$ Cf. RuI DE PINA, Chronica de Affonso V, ed. Almeida (cf. nota 11), p. 759.

${ }^{29} \mathrm{Cf}$. Raymond DE Roover, The Rise and Decline of the Medici Bank, 1397-1494, Cambridge (Mass.), 1963, que no menciona ninguna dependencia del banco en España; cf. las indicaciones no muy precisas, p. 276.

${ }^{30} \mathrm{Cf}$. NASCIMENTO, Leonor de Portugal (cf. nota 2), pp. 34, 48 y 50. Sobre el culto de San Vicente cf. recientemente Sofia SEEGER, Der Vinzenz-Kult im Königreich Portugal unter Afonso Henriques (1128/1139-1185): Ein Kult zur Stütze des neuen Königtums?, Erlangen, 2000 (Magisterarbeit).

${ }^{31}$ Cf. NASCIMENTO, Leonor de Portugal (cf. nota 2), p. 36.

${ }^{32}$ Cf. NASCimento, Leonor de Portugal (cf. nota 2), p. 24. 
con las festividades de esa fiesta y se extrañó de que Lanckmann no las describiera $^{33}$. A mi parecer, no debió de darse inicio a tales festividades antes de la víspera del 25 de julio o de ese día mismo. La temprana partida de Santiago es aún más llamativa, si se piensa que el año del viaje, 1451, era año santo compostelano. El grupo de irlandeses al cual Lanckmann y Motz se unieron quizás llegaran por esa fecha para asistir a las festividades del 25 de julio, a diferencia de los dos sacerdotes alemanes.

Respecto a la apresurada partida de Santiago, se puede formular la siguiente hipótesis: Lanckmann y Motz tenían prisa, puesto que llevaban retraso. Rui de Pina menciona que Afonso V de Portugal ya se había trasladado el 23 de junio a Lisboa con su corte, a la espera de los dos embajadores $^{34}$.

Cuando llegaron a Portugal, la vestimenta de Lanckmann y Motz correspondía más bien a la de peregrinos que a su condición de embajadores del rey de Alemania. Al respecto, se puede decir que Federico III tenía la reputación de ser avaro. También es posible, empero, que con tal indumentaria quisieran evitar asaltos en el camino o que ella tuviera que ver con el robo de que fueron víctimas en el Rabanal ${ }^{35}$. Este imprevisto los dejó sin dinero, situación que sólo palió en parte el préstamo del conde en Ponferrada. Rui de Pina refiere de la siguiente manera el encuentro de Afonso Nogueira, en aquel entonces obispo de Coimbra, con los dos enviados, en la capilla de Santa María: el obispo se encontraba en peregrinación a Santiago, pero al ver el estado calamitoso de los dos forasteros, volvió con ellos a Coimbra, donde los acogió con gran liberalidad, preocupándose, además, de mandarles hacer trajes de fina tela ${ }^{36}$.

Si hemos de creer a Ilja Mieck ${ }^{37}$, el culto a Santiago ya había decaído en importancia en la segunda mitad del siglo XV, es decir antes de los

\footnotetext{
${ }^{33}$ Cf. NASCimento, Leonor de Portugal (cf. nota 2), p. 25 con nota 6.

${ }^{34} \mathrm{Cf}$. RUI DE PINA, Chronica de Affonso $V$, ed. Almeida (cf. nota 11), p. 759, quien añade: $E$ os Embaaxadores do Emperador que eram dous, tardavam ja mais tempo do que fora concordado...

${ }^{35}$ Ésa es la causa que indica RUI DE PINA, Chronica de Affonso V, ed. AlmEIDA (cf. nota 11), pp. $759-760$

${ }^{36}$ Cf. Rui de Pina, Chronica de Affonso V, ed. Almeida (cf. nota 11), pp. 759-760.

${ }^{37} \mathrm{Cf}$. Ilja MIECK, Zur Wallfahrt nach Santiago de Compostela zwischen 1400 und 1650. Resonanz, Strukturwandel und Krise, en "Spanische Forschungen der Görresgesellschaft", Serie I: "Gesammelte Aufsätze zur Kulturgeschichte Spaniens“ 29, Münster, 1978, pp. 483-533.
} 
tiempos de la Reforma. Esta situación tal vez explique, en parte, el poco interés por el 25 de julio y el año santo compostelano de que dieron muestra Lanckmann y Motz. Si echamos una mirada a las cifras de peregrinos ingleses que llegaron a las costas gallegas en barco, con ocasión de los años santos, llama la atención el gran número de peregrinos en 1434 y 1445 y la considerable baja en el número de éstos a partir de 1451 y 1456.

Cifras de peregrinos ingleses ${ }^{38}$

\begin{tabular}{||l|l|l|l|l|l|l|l||}
\hline & $1413:$ & 4 & 180 & + & $1445:$ & 29 & 2100 \\
\hline & $1414:$ & 3 & 140 & + & $1451:$ & 11 & 594 \\
\hline & $1415:$ & 1 & 24 & & $1455:$ & 1 & 50 \\
\hline+ & $1423:$ & 1 & 60 & + & $1456:$ & 15 & 870 \\
\hline+ & $1428:$ & 13 & 916 & & $1474:$ & 1 & 4 \\
\hline & $1432:$ & 1 & 24 & & $1475:$ & 2 & 120 \\
\hline+ & $1434:$ & 64 & 3110 & & & & \\
\hline \hline
\end{tabular}

\section{CONCLUSIÓN}

A modo de conclusión, quisiera resaltar la diferencia entre el relato de Lanckmann y el de otros viajeros, puesto que el primero sólo trata al margen algunas tradiciones jacobeas. En ese sentido, Andreas Meyer ya ha hecho notar la poca atención que los peregrinos del siglo XV prestaban a la tumba del Apóstol en Santiago ${ }^{39}$.

\footnotetext{
${ }^{38}$ En la primera columna, aparece el año (la cruz indica que se trata de un año santo compostelano, esto es, que el 25 de julio cae en un día domingo); siguen la cantidad de barcos con peregrinos (vale decir, la cantidad de licencias) y el número de peregrinos. Cf. MIECK, Santiago-Wallfahrt (cf. nota 37), p. 489.

${ }^{39} \mathrm{~A}$ finales del siglo XV, se habría hecho más frecuente la creencia que el cuerpo del Santo reposaba en Tolosa y no en Santiago de Compostela. Cf. Andreas MEYER, Städtische Identität und Konkurrenz. Die spätmittelalterlichen Apostelgräber in Toulouse, en "Stadt und Pilger. Soziale Gemeinschaften und Heiligenkult auf der Iberischen Halbinsel", ed. Klaus HERBERS, Tubinga, 1999 ("Jakobus-Studien" 10), pp. 125-140, esp. pp. 138-139, y Andreas MEYER, Von
} 
El texto analizado muestra que el intercambio cultural entre pueblos diferentes no tan sólo se produce por medio de la exogamia - estudiada por Karl-Heinz $\mathrm{Spie}^{40}{ }^{40}$, entre otros investigadores- sino también de las peregrinaciones. La importancia de esta fuente radica en que fue traducida al alemán, impresa y utilizada por autores de otras obras de comienzos del siglo XVI - por ejemplo por el autor del Weißkunig-, las cuales encontraron gran difusión ${ }^{41}$. Las peripecias del viaje a Portugal, como el asalto a mano armada, que Lanckmann plasmó en su relato, pudieron, de este modo, ser conocidas por un gran círculo de lectores e influir en la idea que la gente en Alemania se hacía de la peregrinación jacobea y de la Península Ibérica.

Santiago de Compostela nach Toulouse. Ein Apostel verlegt sein Grab, "Francia", $26 / 1$ (1999), pp. 209-238, esp. p. 214.

${ }^{40} \mathrm{Cf}$. SPIEß, Unterwegs (cf. nota 7).

${ }^{41}$ Sobre impresores alemanes en Portugal, cf. Marion EHRHARDT, Frühe deutsche Drucker in Portugal, en MARQUES/OPITZ, "Portugal-Alemanha" (cf. nota 9), pp. 25-30. 


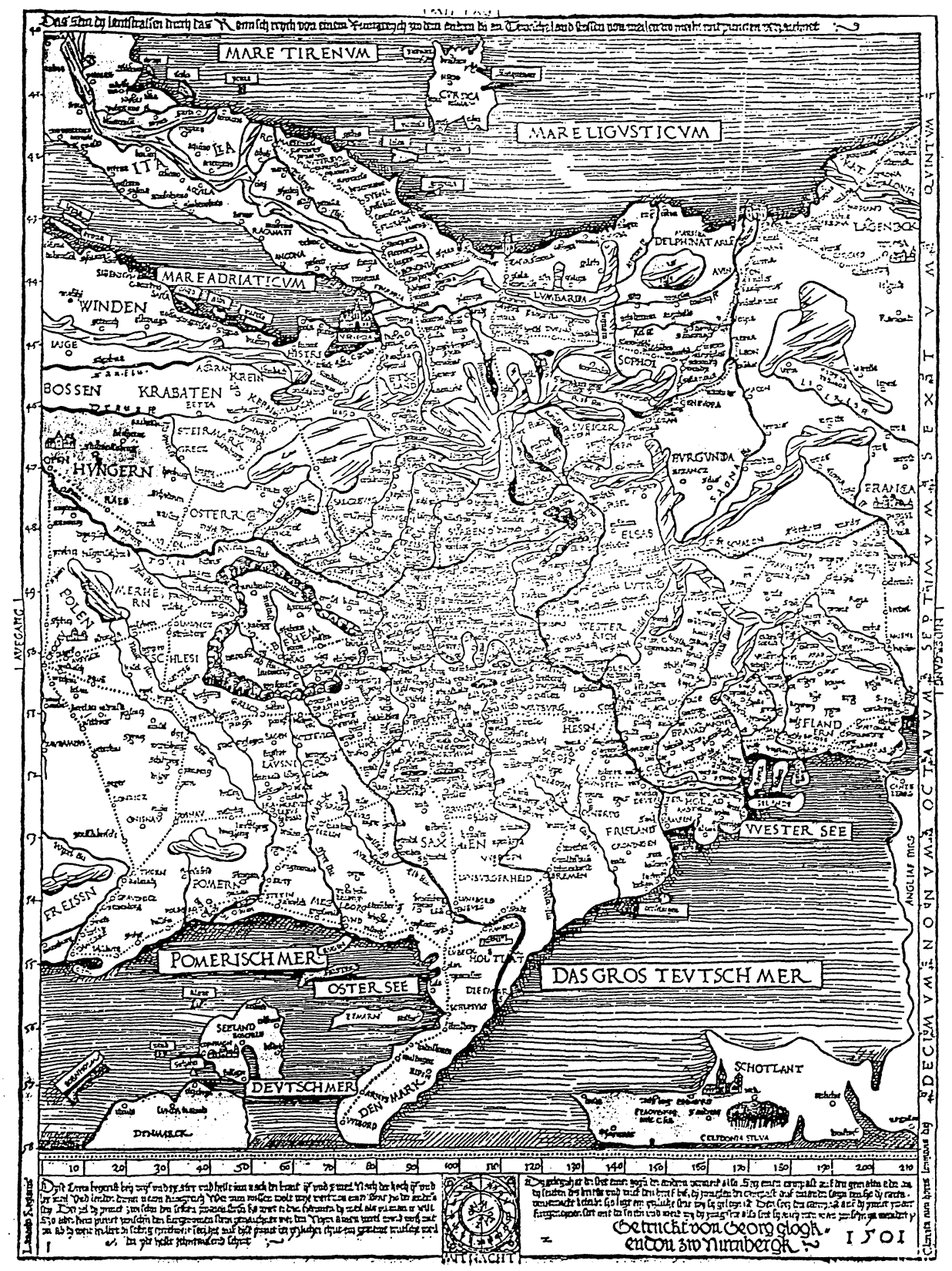

Mapa de Centroeuropa Erhard Etzlaub, Nurenberg 1501. 


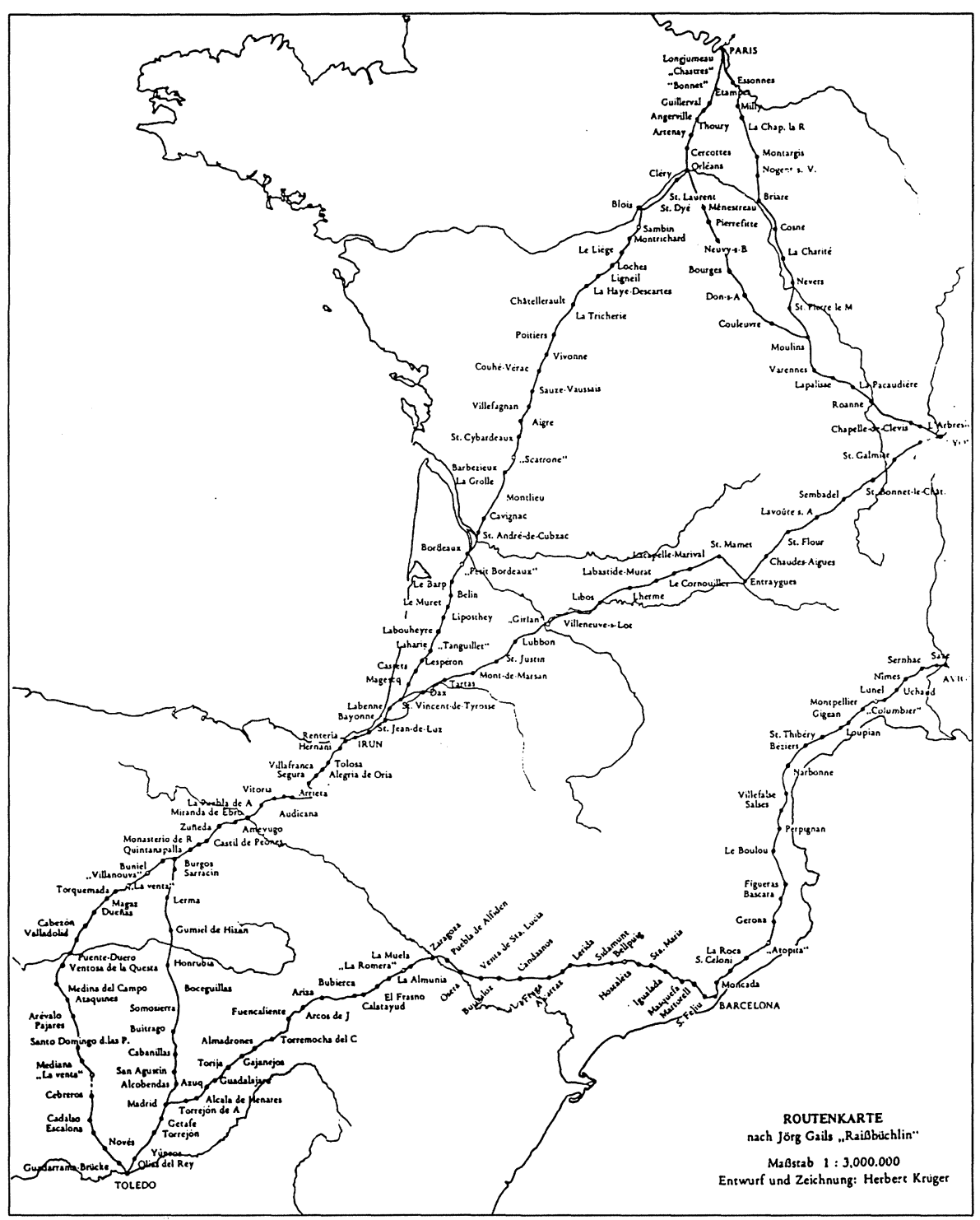

Rutas hacia el sud-oeste de Europa, según el "Raissbuechlin" de Joerg Gail, Augsburg 1563; mapa: H. Krüger. 